\title{
Mixtures of foam and paste: suspensions of bubbles in yield stress fluids
}

\author{
Michael Kogan · Lucie Ducloué · Julie Goyon · Xavier Chateau · Olivier Pitois · \\ Guillaume Ovarlez
}

Received: date / Accepted: date

\begin{abstract}
We study the rheological behavior of mixtures of foams and pastes, which can be described as suspensions of bubbles in yield stress fluids. Model systems are designed by mixing monodisperse aqueous foams and concentrated emulsions. The elastic modulus of the bubble suspensions is found to depend on the elastic capillary number $C a_{G}$, defined as the ratio of the paste elastic modulus to the bubble capillary pressure. For values of $C a_{G}$ larger than $\simeq 0.5$, the dimensionless elastic modulus of the aerated material decreases as the bubble volume fraction $\phi$ increases, suggesting that bubbles behave as soft elastic inclusions. Consistently, this decrease is all the sharper as $C a_{G}$ is high, which accounts for the softening of the bubbles as compared to the paste. By contrast, we find that the yield stress of most studied materials is not modified by the presence of bubbles. This suggests that their plastic behavior is governed by the plastic capillary number $C a_{\tau_{y}}$, defined as the ratio of the paste yield stress to the bubble capillary pressure. At low $C a_{\tau_{y}}$ values, bubbles behave as nondeformable inclusions, and we predict that the suspension dimensionless yield stress should remain close to unity, in agreement with our data up to $C a_{\tau_{y}}=0.2$. When preparing systems with a larger target value of $C a_{\tau_{y}}$, we observe bubble breakup during mixing, which means that they have been deformed by shear. It then seems that a critical value $C a_{\tau_{y}} \simeq 0.2$ is never exceeded in the final material. These observations might imply that, in bubble suspensions prepared by mixing a foam and a paste, the suspension yield stress is always close to that of the paste surrounding the bubbles. Finally, at the highest $\phi$ investigated, the yield stress is shown to increase abruptly with $\phi$ : this is interpreted as a 'foamy yield stress fluid' regime, which takes place when the paste mesoscopic con-
\end{abstract}

Université Paris-Est, Laboratoire Navier (UMR CNRS 8205), Champssur-Marne, France, E-mail: guillaume.ovarlez@ifsttar.fr stitutive elements (here, the oil droplets) are strongly confined in the films between the bubbles.

Keywords Yield stress fluid - Bubbles - Suspension · Foam $\cdot$ Emulsion $\cdot$ Elastic modulus $\cdot$ Yield stress

\section{Introduction}

Many dense suspensions involved in industrial processes (concrete casting, drilling muds, foodstuff transport...) and natural phenomena (debris-flows, lava flows...) are yield stress fluids (Coussot 2005). These are usually very polydisperse systems in which yield stress arises from the colloidal forces between the smallest particles (Mewis and Wagner 2012), and is increased by the presence of rigid noncolloidal particles (Mahaut et al. 2008a; Chateau et al. 2008).

In addition, these materials often contain air bubbles. This is the case, e.g., of crystal bearing magmas (Griffiths 2000; Gonnermann and Manga 2007), or of aerated food emulsions (van Aken 2001). In industry, gas is typically injected into materials to improve their performance or to make them lighter. The fact that the latter materials exhibit a yield stress is essential to prevent bubbles from rising (Dubash and Frigaard 2004, 2007; Sikorski et al. 2009). Besides stability issue, it is particularly important to understand the mechanical behavior of these aerated materials. In many processes, gas incorporation is obtained by mixing a foam with a paste. This is the case, e.g., in plasterboard production, in which a foamed plaster slurry is prepared by mixing an aqueous foam with a gypsum plaster slurry, in foam concrete production by pre-foaming methods, where aqueous foam is mixed with a base mix (Ramamurthy et al. 2009), or in food processing when mixing beaten egg whites and a batter to make aerated food products. It is thus crucial to understand the impact of mixing a foam with a paste, and more generally of adding bubbles to a yield stress fluid. 
Even the simpler case of bubbles in viscous fluids has The case of rigid particles in yield stress fluids has been been the subject of only a few experimental studies Rust and Mainatestigated recently in several studies (Ancev and Jorrot 2001; 2002a. b; Llewelin et al. 2002). It has been shown that the viscosity of suspensions of bubbles in a Newtonian fluid depends on the applied shear stress $\tau$, and is characterized by two different viscosity plateaus at low and high values of $\tau$ (Rust and Manga 2002a). At low applied stress, the viscosity of the suspension is an increasing function of the bubble volume fraction $\phi$; at high values of $\tau$, it decreases with $\phi$. This phenomenon is attributed to interfacial phenomena and to the ability of the bubbles to resist shear-induced deformation (Rust and Manga 2002b). At low applied stress, bubbles are not deformed; they behave like rigid spherical particles with a slip boundary condition, thus explaining the viscosity increase with $\phi$. Above a critical stress, surface tension is no longer sufficient to maintain the bubbles in a spherical shape; the bubbles are deformed by shear, which leads to a decrease of the viscosity with $\phi$. This transition from nondeformable to deformable bubbles has indeed been shown to be driven by a capillary number, which compares the shear stress and the interfacial stress Rust and Manga 2002a). More details are given in Sec.2.2

Most studies of bubbles in yield stress fluids have focused on the stability of these systems against coarsening and buoyancy, both in specialized fields (Dutta et al. 2004a. b; Lev et al. 2009) and fundamental studies (Koczo et al. 1992; Turner et al. 1999; Dubash and Frigaard 2004, 2007; Sikorski 2009; Goyon et al. 2010; Salonen et al. 2012). To our knowledge, the impact of bubbles on the rheological properties of such systems has been studied only for specific materials, but has not been the subject of fundamental studies. Struble and Jiang (2004) have studied the effect of air entrainment on cement pastes and concrete rheology. In these materials, an air-entraining agent (a surfactant) is put in the paste; this stabilizes the bubbles that are produced by agitating the paste. Rheological measurements show an increase of the material yield stress with the air bubble fraction, whereas its plastic viscosity decreases; this is a priori surprising because, according to the results of Rust and Manga (2002a), the first observation would imply that bubbles are not deformed by shear whereas the second would imply that they are deformed. It is worth noting that the bubbles obtained with air-entraining agents are likely to be very polydisperse. This size dispersity is not controlled, which poses at least two problems: (i) scale separation between the bubbles and the cement particles is not ensured, which may not allow the performance of classical micromechanical analyses by considering the interstitial paste as a continuous medium, and (ii) in some conditions, the largest bubbles may be deformable under shear whereas the smallest are not, which can make the overall response rather complex. Finally, it appears that the role of bubbles in the rheology of yield stress fluids has yet to be fully understood.

Geiker et al. 2002a; Mahaut et al. 2008a,b; Vu et al. 2010). Mahaut et al. (2008a) have performed studies on model suspensions of monodisperse particles in various yield stress fluids (concentrated emulsions, Carbopol gels, colloidal gels). They have observed that their elastic modulus $G^{\prime}(\phi)$ increases with the particle volume fraction $\phi$, and is well fitted to a Krieger-Dougherty equation, as classically observed for suspensions of particles in linear materials. Their yield stress $\tau_{y}(\phi)$ increases more moderately with $\phi$. Mahaut et al. (2008a) have shown that the dimensionless elastic modulus $G^{\prime}(\phi) / G^{\prime}(0)$ and the dimensionless yield stress $\tau_{y}(\phi) / \tau_{y}(0)$ of these systems are related through a simple relationship with no fitting parameter, as predicted by Chateau et al. (2008), thus leading to a simple theoretical expression for $\tau_{y}(\phi)$ in agreement with experimental data (more details are given in Sec. 2.1. It is finally sufficient to characterize only one rheological property of these materials to predict the value of the other ones. These results, obtained on model systems, have been shown to be applicable to more complex systems such as model mortars made of rigid spherical particles in cement pastes (Mahaut et al. 2008b).

A question that arises is how these results are changed ahnen rigid particles are replaced by gas bubbles, and what the role of bubble deformability is on the rheological behavior of suspensions in yield stress fluids. In particular, by analogy with suspensions of bubbles in Newtonian fluids, we need to understand how the deformability of bubbles is controlled when the material is sheared. We also question the possible link between the linear and nonlinear properties of these systems.

In this paper, we investigate the rheological behavior of suspensions of bubbles in yield stress fluids. In Sec. 2 we briefly review and discuss the theoretical behavior of suspensions of rigid particles and bubbles in linear (elastic or viscous) and nonlinear (plastic) materials. We deal in particular with the issue of bubble deformability in sheared yield stress fluids. In Sec. 3 we present the model systems used in this work, made by mixing a monodisperse foam and a model yield stress fluid, namely a concentrated emulsion. The experimental results are presented in Secs.4 and[5 We first study the change in the elastic properties of the yield stress fluid in its solid regime as the foam fraction added to the material (and thus bubble volume fraction) is progressively increased (Sec. 4). We then study the evolution of the plastic properties (Sec. [5). The study of viscoplastic properties is reserved for future work. 


\section{Theory}

In the following, we consider monodisperse 1 spherical inclusions of diameter $d$ dispersed in materials at a volume fraction $\phi$. When they are dispersed in a linear material, namely a Hookean material of elastic shear modulus $G^{\prime}(0)$ or a Newtonian material of viscosity $\eta(0)$, the property of interest is the dimensionless linear response of the material $g(\phi)$ (i.e., the dimensionless elastic modulus $G^{\prime}(\phi) / G^{\prime}(0)$ or the dimensionless viscosity $\eta(\phi) / \eta(0))$. When they are dispersed in a plastic material of yield stress $\tau_{y}(0)$, this becomes the value of the dimensionless yield stress $\tau_{y}(\phi) / \tau_{y}(0)$.

In this section, we first review the case of suspensions of solid rigid inclusions (particles), which has already been thoroughly investigated in the literature, and provides a solid framework to better understand the behavior of deformable inclusions. We then discuss the possible behavior of suspensions of gas inclusions (bubbles), which is not yet well understood.

\subsection{Suspensions of rigid particles}

We first focus on suspensions of rigid particles. Particles are considered as perfectly rigid, and a no-slip boundary condition is assumed at the interface between the particles and the suspending material.

\section{Linear response}

In the dilute limit $(\phi<<1)$, it is shown that Larson 1999)

$g(\phi)=1+2.5 \phi$

Many theoretical expressions exist for values of $\phi$ beyond the dilute limit (Stickel and Powell 2005). For isotropic suspensions, it is shown that $g(\phi)$ should be higher than the Hashin-Shtrikman bound (Hashin and Shtrikman 1963) for any value of $\phi$ :

$g(\phi) \geq \frac{1+\frac{3}{2} \phi}{1-\phi}$

Experimentally, the linear behavior of suspensions is usually found to be consistent with the Krieger-Dougherty phenomenological equation (Stickel and Powell 2005)

$g(\phi)=\frac{1}{\left(1-\phi / \phi_{\mathrm{div}}\right)^{2.5 \phi_{\mathrm{div}}}}$

which complies with Eq. 1 in the dilute limit. A value of $\phi_{\text {div }}=0.57$ was found experimentally for isotropic suspensions (Mahaut et al. 2008a), and $\phi_{\text {div }}=0.605$ for anisotropic suspensions (structured by a flow) (Ovarlez et al. 2006).

\footnotetext{
1 Some aspects of the linear and nonlinear behavior of polydisperse suspensions are discussed in (Vu et al. 2010).
}

Nonlinear response

Chateau et al. (2008) have developed a simple micromechanical approach to predict the nonlinear behavior of suspensions in yield stress fluids. The starting point is that, if the particles do not store any elastic energy (rigid limit), the local strain in the interstitial material $\gamma_{\text {local }}(\phi)$ when a given strain $\Gamma_{\text {macro }}$ is applied to the suspension can be estimated at first approximation as

$\gamma_{\text {local }}(\phi)=\Gamma_{\text {macro }} \sqrt{g(\phi) /(1-\phi)}$

This information on the local strain can be used to predict the value of the dimensionless yield stress of the suspension, which finally implies that it is related to its dimensionless elastic modulus through a simple relationship with no fitting parameter (Chateau et al. 2008):

$\tau_{y}(\phi) / \tau_{y}(0)=\sqrt{(1-\phi) g(\phi)}$

In the dilute limit, the dimensionless yield stress is thus obtained by combining Eqs. 1 and 5 .

$\tau_{y}(\phi) / \tau_{y}(0)=1+\frac{3}{4} \phi$

Combining Eqs. 3 and 5 finally yields a simple phenomenological law

$\tau_{y}(\phi) / \tau_{y}(0)=\sqrt{(1-\phi)\left(1-\phi / \phi_{\text {div }}\right)^{-2.5 \phi_{\text {div }}}}$

which is in good agreement with the observations of Mahaut et al. (2008a) for $\phi \leq 50 \%$, thus validating Eqs. 4 and 5

Similarly, when the interstitial yield stress fluid behaves as a Herschel-Bulkley material $\tau=\tau_{y}+\eta_{H B} \dot{\gamma}^{n}$, it is shown that a simple relationship exists between the dimensionless yield stress and the dimensionless consistency $\eta_{H B}(\phi) / \eta_{H B}(0)$ (Chateau et al. 2008).

\subsection{Suspensions of bubbles}

\section{Linear response}

For suspensions of bubbles in linear materials, two limiting cases are well known: that of nondeformable bubbles (with infinite surface tension to bubble diameter ratio), and that of fully deformable bubbles (with no surface tension). In all cases, a slip boundary condition is assumed at the interface between the bubbles and the interstitial material.

For nondeformable bubbles, the linear response in the dilute limit is (Larson 1999)

$g(\phi)=1+\phi$

The difference with rigid particles (Eq. 1) comes from the slip boundary condition at the bubble interface. For fully deformable bubbles, in the dilute limit, $g(\phi)$ is

$g(\phi)=1-\frac{5}{3} \phi$ 
Little is known about the behavior at higher $\phi$. Theoretical bounds nevertheless exist. For isotropic suspensions of fully deformable bubbles, $g(\phi)$ should be lower than the MoriTanaka bound (Dormieux et al. 2006) at any $\phi$

$g(\phi)<\frac{1-\phi}{1+\frac{2}{3} \phi}$

Overall, $g(\phi)$ is expected to increase with $\phi$ for nondeformable bubbles and to decrease with $\phi$ for fully deformable bubbles, consistent with the observations of Rust and Manga (2002a). Between these two limiting cases, the deformability of bubbles should depend on the resistance offered by the bubbles to shear-induced deformation, due to the air/fluid surface tension $\sigma_{t}$. The relevant dimensionless number involved in the bubble deformability should then be a capillary number $\mathrm{Ca}$, constructed by comparing the characteristic stress $4 \sigma_{t} / d$ due to surface tension to a characteristic applied shear stress.

For bubbles suspended in a Newtonian fluid of viscosity $\eta_{0}$ sheared at a shear rate $\dot{\gamma}$, the only characteristic shear stress is $\eta_{0} \dot{\gamma}$. It has indeed been shown (Rust and Manga 2002a) that

$C a_{\eta}=\frac{\eta_{0} \dot{\gamma}}{2 \sigma_{t} / d}$

drives the transition between nondeformable bubbles (viscosity increasing with $\phi$ ) and deformable bubbles (viscosity decreasing with $\phi$ ). This transition occurs at $C a_{\eta} \approx 1$.

To our knowledge, no rigorously derived theoretical expression valid for all values of $C a_{\eta}$ exists in the literature. The full time-dependent tensorial behavior of suspensions of viscous drops has been calculated by Frankel and Acrivos (1970) in the dilute limit, in the framework of a first-order perturbation analysis: drops are assumed to be only slightly deformable. In the case of suspensions of bubbles, this theory applies only at low $C a_{\eta}$ and is written as a first-order expansion in $C a_{\eta}$. For steady-state flows in simple shear, the dimensionless viscosity reduces to Eq. 8, it does not depend on $\mathrm{Ca} a_{\eta}$ at order 1 ; in addition, normal stress differences proportional to $C a_{\eta}$ are predicted: we will not discuss this aspect in this paper. Several works Rust and Manga 2002a; Llewelin et al. 2002; Pal 2004) have proposed phenomenological equations intended to be valid at any values of $C a_{\eta}$ and $\phi$. Their starting point is Frankel and Acrivos (1970) equation, with the additional strong assumption that this equation can be used at any value of $C a_{\eta}$. Some of these expressions have been shown to fit reasonably well experimental data; in Sec.4.3. we will use the best fitting function to experimental data proposed by Rust and Manga (2002a) and $\mathrm{Pal}$ (2004) for comparison with our data.

The case of bubbles with surface tension in elastic materials has not been studied to our knowledge. Two capillary numbers can a priori be built to describe the behavior of such materials, one based on the elastic modulus of the material $G^{\prime}(0)$ :

$$
C a_{G}=\frac{G^{\prime}(0)}{2 \sigma_{t} / d}
$$

and one based on the applied stress $\tau=G^{\prime}(0) \Gamma$ (where $\Gamma$ is the applied strain) during an elastic modulus measurement:

$$
C a_{\tau}=\frac{G^{\prime}(0) \Gamma}{2 \sigma_{t} / d}
$$

For the materials we study (Sec. 3.1): $2 \sigma_{t} / d$ ranges between 25 and $1000 \mathrm{~Pa}, G^{\prime}$ is typically of order 100 to $1000 \mathrm{~Pa}$, and the applied stress during an elastic modulus measurement is typically of order $1 \mathrm{~Pa}$ or less. The two capillary numbers defined above would thus lead to descriptions of very different behaviors. On the one hand, $C a_{\tau}$ is of order 0.01 for all studied systems, which would imply that bubbles should behave as nondeformable objects; on the other hand, $C a_{G}$ is of order unity, which would imply that a transition between the behavior of a suspension of nondeformable bubbles and that of a suspension of deformable bubbles should be observed. In our experiments, by characterizing the elastic moduli of suspensions of various compositions, we thus expect to be able to identify the relevant capillary number.

\section{Nonlinear response}

Not much is known about the plastic behavior of suspensions of bubbles in plastic materials. For nondeformable and fully deformable bubbles, no elasticity is stored in the interfaces. Eqs. 4 and 5 are thus expected to remain valid. Combining Eq. 5] with Eqs. 8 and 9, it is thus predicted that, in the dilute limit, the dimensionless yield stress of suspensions of nondeformable bubbles should be

$\tau_{y}(\phi) / \tau_{y}(0)=1$

whereas the dimensionless yield stress of suspensions of fully deformable bubbles should be

$\tau_{y}(\phi) / \tau_{y}(0)=1-\frac{4}{3} \phi$

At this stage, as elasticity is stored in the interfaces for intermediate cases, we cannot tell anything about the value of the dimensionless yield stress and about its possible link with $g(\phi)$ between these two limiting cases. In the case of plastic flows, the characteristic stress is the interstitial material yield stress $\tau_{y}$ and the capillary number that should drive the transition from nondeformable to deformable bubbles is expected to be

$C a_{\tau_{y}}=\frac{\tau_{y}}{2 \sigma_{t} / d}$ 


\section{Materials and methods}

\subsection{Materials}

\section{Principle}

Model suspensions of bubbles in a yield stress fluid are prepared by mixing a monodisperse foam and a concentrated emulsion. The emulsion is made of oil droplets dispersed at a high volume fraction (typically $82 \%$ ) in an aqueous surfactant solution; this is a simple yield stress fluid (Mason et al. 1996; Ovarlez et al. 2008). The foam is a dispersion of monodisperse bubbles at a high volume fraction (typically 90\%) in an aqueous surfactant solution of the same composition as that in the concentrated emulsion. The similarity of the continuous phases of both systems ensures (i) that the two materials are easily mixed together, and (ii) that after mixing, the bubbles are surrounded by a known yield stress fluid. This interstitial fluid is a concentrated emulsion of droplet volume fraction slightly lower than that of the initial emulsion, which has been diluted by the aqueous solution brought in by the foam. All systems are such that (i) the droplet volume fraction is high enough in the final interstitial emulsion so that it has a yield stress, and that (ii) the bubble diameter is much larger than the droplet diameter to ensure that the emulsion is 'seen' as a continuous material (a yield stress fluid) by the bubbles.

\section{Emulsion preparation}

Batches of 21 of oil-in-water emulsion are prepared by dispersing dodecane (Acros Organics) at $82 \%$ or $85 \%$ volume fraction in a surfactant solution (see below) with a Silverson L4RT mixer. The rotation speed is kept at $1000 \mathrm{rpm}$ during the addition of dodecane. It is then increased to $6000 \mathrm{rpm}$ during $\approx 30$ min until a homogeneous emulsion is formed. The average droplet diameter in the final emulsion is $4.2 \mu \mathrm{m}$; the polydispersity (computed as in (Mabille et al. 2000) is of order $20 \%$. The viscosity of dodecane is $\eta_{o}=1.3 \mathrm{mPa} . \mathrm{s}$ at $25^{\circ} \mathrm{C}$.

\section{Foam}

Monodisperse foams are produced by either blowing air through a porous glass frit or through a needle into a glass syringe filled with a surfactant solution. The needle tip was cut and crushed: depending on the way the tip was crushed, a different bubble size was obtained for each needle; in the case of the porous frits, different bubble sizes were obtained depending on the pore size. In both cases, the air was blown at a flow rate of the order of a few $\mathrm{ml} / \mathrm{min}$ (depending on the bubble size), and was saturated with perfluorohexane vapor $\left(\mathrm{C}_{6} \mathrm{~F}_{14}\right.$, Sigma-Aldrich), which strongly reduces the bubble coarsening rate (Gandolfo and Rosano 1997). We make monodisperse foams of bubble diameter varying between $110 \mu \mathrm{m}$ and $3 \mathrm{~mm}$ (with a size dispersity of order $3 \%$ ), which is much larger than the emulsion droplet diameter. The size of the bubbles was checked by squeezing out a small quantity of the foam into a petri dish filled with surfactant solution and examining them under an upright microscope. Slightly polydisperse foams of $100 \pm 30 \mu \mathrm{m}$ bubble diameter were also produced by blowing air through a porous medium made of a glass bead assembly: this setup allows us to produce a large volume of foam made of small bubbles in a reasonable time, that is, before coarsening occurs.

\section{Surfactant solutions}

The surfactant solutions used in these studies are made of sodium dodecyl sulfate surfactant (SDS, Sigma-Aldrich) dispersed in deionized water at a $28 \mathrm{~g} / 1$ concentration in the emulsions, and $5 \mathrm{~g} / \mathrm{l}$ in the foams. The air/surfactant solution surface tension $\sigma_{t}$ was measured with the pendant drop method; its value is $0.036 \pm 0.001 \mathrm{~N} . \mathrm{m}^{-1}$. The surfactant concentration has been chosen to be high enough to saturate the oil droplet and bubble interfaces. Note that the critical micelle concentration (CMC) of the SDS solution is $2.3 \mathrm{~g} / 1$.

Complementary experiments have been performed with two other surfactant solutions (both in the emulsion and the foam) in order to study the role of surface mobility (Denkov et al. 2009): TetradecylTrimethylAmmonium Bromide (TTAB) and a TTAB solution with dodecanol. Those systems exhibited the same behavior as those prepared with SDS; in this paper, we thus present only the results obtained in systems prepared with the SDS surfactant.

\section{Preparation of suspensions of bubbles}

Two kinds of studies are performed. First, we investigate the evolution of the rheological behavior of a yield stress fluid when foam is added to the material (this matches what may happen in an industrial process). In a first series of experiments, samples are thus prepared by mixing a foam and an emulsion in various mass ratios (Fig. 11). In this case, as the foam fraction is progressively increased, two changes occur in the final material: (i) the bubble volume fraction increases, and (ii) the droplet volume fraction in the interstitial emulsion decreases (due to the additional surfactant solution brought in by the foam). To understand the impact of adding bubbles to a yield stress fluid, the properties of the bubble suspension have to be compared to that of the yield stress fluid surrounding the bubbles. For each sample, we thus also prepare a pure emulsion sample by adding to the initial emulsion a mass of surfactant solution equal to the mass of the foam added to make the suspension sample. This 


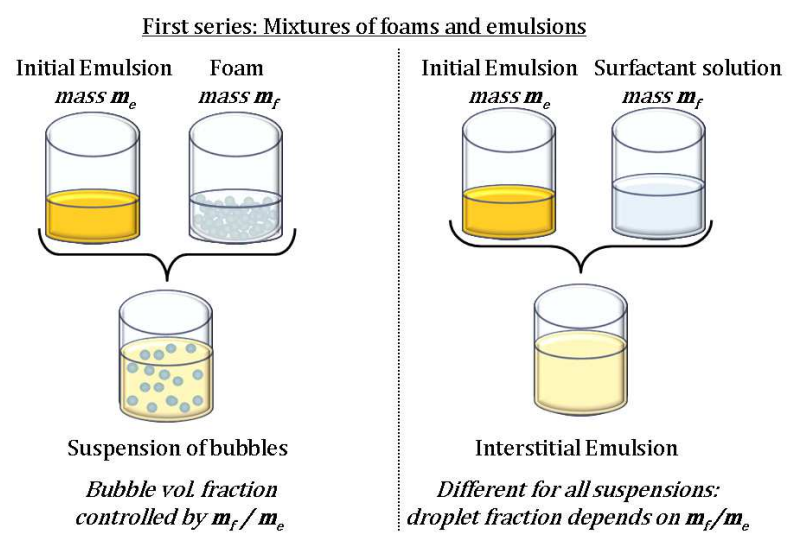

Second series: Bubble suspensions of same interstitial emulsion Initial Emulsion Foam Surfactant solution Initial Emulsion Surfactant solution mass $\boldsymbol{m}_{c}$ Suspension of bubbles Bubble vol. fraction controlled by $\boldsymbol{m}_{f} /\left(\boldsymbol{m}_{f}+\boldsymbol{m}_{s}+\boldsymbol{m}_{e}\right)$

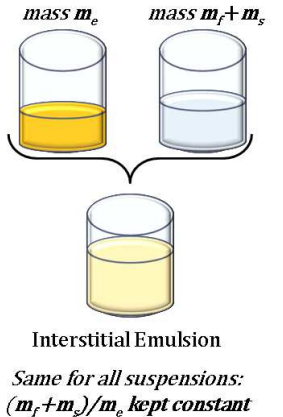

Fig. 1 Sketch of the material preparation in the two series of experiments.

allows us to characterize the interstitial emulsion for each prepared suspension. In this series of experiments, the elastic modulus of the initial emulsion (before mixing with the foam) is $G^{\prime}=625 \mathrm{~Pa}$ and its yield stress is $\tau_{y}=35 \mathrm{~Pa}$. The bubble volume fraction $\phi$ is varied between $0 \%$ and $90 \%$.

In a second series of experiments, we focus on the role of bubbles by studying the impact of increasing the bubble volume fraction in an interstitial yield stress fluid of constant properties (Fig. 1 b). To this end, materials are prepared by adding various mixes of foam (mass $m_{f}$ ) and surfactant solution ( $\operatorname{mass} m_{s}$ ) to the emulsion (mass $m_{e}$ ). The ratio $\frac{m_{f}+m_{s}}{m_{e}}$ is kept constant, which ensures that the final droplet volume fraction in the emulsion surrounding the bubbles is always the same. The bubble volume fraction in the material is then changed by varying $m_{f} / m_{s}$. All suspensions are compared to the interstitial emulsion, which is made with the same recipe, with $m_{f} / m_{s}=0$. In this series of experiments, the elastic modulus of the interstitial emulsion is $G^{\prime} \approx 280 \mathrm{~Pa}$ and its yield stress is $\tau_{y} \approx 9 \mathrm{~Pa}$. A few experiments with the largest bubbles are also performed with interstitial emulsions of yield stress equal to 19 and $72 \mathrm{~Pa}$ to better test the possible deformability of bubbles under shear. The bubble volume fraction $\phi$ is here varied between $0 \%$ and $60 \%$.
The foam, surfactant solution, and the emulsion are mixed together in a $9.5 \mathrm{~cm}$ diameter beaker using a mixer with a $6.8 \mathrm{~cm} \times 6.8 \mathrm{~cm}, 6$-hole rectangular blade. The velocity is initially at $60 \mathrm{rpm}$, and is increased to $100 \mathrm{rpm}$ after $\approx 10 \mathrm{~s}$ of mixing. The mixing then continues until the mixture is homogeneous, which occurs after $\approx 60 \mathrm{~s}$.

The volume fraction of bubbles within the emulsion is estimated from the measurement of the density of the suspension in a petri dish of known volume. The absolute uncertainty on the material volume fraction, accounting for both the measurement uncertainty and the possible volume fraction spatial variations in a given sample, is 0.02 . We disregarded the few samples where the experimentally measured bubble fraction was greater than that expected from the foam fraction in the mixture, to ensure that no unwanted bubbles were added to the material.

All systems are designed to be stable at rest: their yield number $Y=\frac{\tau_{y}}{\Delta \rho g d}$ complies with the stability criterion of a single bubble embedded in a yield-stress fluid (Dubash and Frigaard 2007), which should ensure that elastic forces exerted by the yield stress fluid at rest are able to counterbalance the net gravity force.

We checked on several prepared suspensions that (i) this procedure leads to a homogeneous material, by measuring the density of several samples extracted from different heights in a given material, and that (ii) the bubble size in the suspension is equal to that in the initial foam, i.e., there is neither shear-induced coalescence nor breakup during mixing, except in the cases discussed at the end of Sec. 5, and no significant bubble coarsening occurs at the time scale of the experiments.

\subsection{Rheometry}

Rheometric experiments are performed within a vane-in-cup geometry (dimensions for bubbles of diameter $d \leq 320 \mu \mathrm{m}$ : inner radius $R_{i}=12.5 \mathrm{~mm}$, outer cylinder radius $R_{e}=18 \mathrm{~mm}$, height $H=46 \mathrm{~mm}$; dimensions for $d=800 \mu \mathrm{m}$ to $d=$ $3 \mathrm{~mm}: R_{i}=22.5 \mathrm{~mm}, R_{e}=45 \mathrm{~mm}, H=46 \mathrm{~mm}$ ) on a commercial rheometer (Bohlin C-VOR 200) that imposes either the torque or the rotational velocity (with a torque feedback). In order to avoid wall slip (Coussot 2005), we use a six-blade vane as an inner tool, and we glue sandpaper on the outer cylinder wall. Working within these widegap geometries allows for study of samples with large bubbles and ensures that, for all the materials studied, there are enough bubbles in the gap to consider that we are measuring the properties of a continuous medium (the suspension). In such a geometry, the shear stress distribution in the gap is heterogeneous. Therefore, one has to choose a definition of the shear stress $\tau$ that is measured in a given rheological experiment. Here, we want to perform both elastic modulus and yield stress measurements. Whatever the 
measurement method we choose, yield first occurs where the stress is maximal i.e. along the inner virtual cylinder delimited by the blades. 2 . As a consequence, the contribution to the torque $T$ of the sheared material at yield is: $\tau_{y} *$ $2 \pi H R_{i}^{2}$; as the material is also sheared at the bottom of the vane, another contribution to the torque is given by $\tau_{y} *$ $2 \pi R_{i}^{3} / 3$ (Dzuy and Boger 1983). We thus define the shear stress measurement as $\tau\left(R_{i}\right)=\frac{T}{2 \pi H R_{i}^{2}\left(1+R_{i} / 3 H\right)}$, so that the yield stress $\tau_{y}$ is correctly measured; this is validated by comparison with measurements performed in a cone-andplate geometry. To ensure correct elastic modulus measurement, as the strain field does not have azimuthal symmetry in the elastic regime, we calibrate the conversion factor between the rotation angle $\Theta$ and the strain $\gamma$ to ensure that the same elastic modulus is measured in a cone-and-plate geometry and in the vane-in-cup geometry; due to the linearity of the behavior, this calibration made in a particular linear elastic material remains valid for any linear elastic material.

\subsection{Procedure, elastic modulus and yield stress measurements}

Before designing the experimental procedure, we need to define precisely the state of the materials we want study. Two points are important: (i) measurements should be performed on a homogeneous suspension, and (ii) the microstructure of the suspensions should be controlled. These points impose severe restrictions on the preparation and the yield stress measurement procedure, as measurements involving an significant flow of suspensions (a large strain) pose several problems.

First, flow causes particle migration (Leighton and Acrivos 1987; Phillips et al. 1992; Ovarlez et al. 2006) towards the low shear zones (the outer cylinder in coaxial cylinder geometries) i.e. creation of a heterogeneous structure; in addition, with a vane tool, particle depletion is induced near the vane blades (Ovarlez et al. 2011) leading to the equivalent of a slip layer. The same might happen with bubbles. In density-mismatched suspensions, even those stable at rest, another source of heterogeneity is gravity (Ovarlez et al. 2010, 2012): bubbles tend to rise when the material is sheared, and rise faster and faster as the shear rate is increased (Goyon et al. 2010). To circumvent these problems, we have decided (i) to avoid any preshear of the material, and (ii) to measure the static yield stress; any other yield stress measurement method based on a shear flow such as shear rate (Geiker et al. 2002b) or shear stress ramps (Uhlherr et al. 2005) and creep tests (Coussot et al. 2006) may lead to heterogeneities. We

\footnotetext{
${ }^{2}$ Note that although flow does not generally have an azimuthal symmetry in a vane-in-cup geometry (Baravian et al. 2002; Ovarlez et al. 2011), azimuthal symmetry seems to be recovered when the shear stress is close to the yield stress Keentok et al. 1985; Ovarlez et al. 2011))
}

have also decided to postpone the study of viscous dissipation in these materials, which would require special care and may require the use of MRI methods to measure local volume fractions (Goyon et al. 2010). Therefore, we here focus on the impact of bubbles on the elastic modulus and the static yield stress of yield stress fluids.

Another problem is that an anisotropic microstructure (distribution of neighbors) is created when suspensions of particles flow Gadala-Maria and Acrivos 1980; Parsi and Gadala-Maria 1987); in addition, with deformable bubbles, bubble orientation is expected to depend on shear history (Rust and Manga $2002 b)$. Suspensions of isotropic and anisotropic microstructure have very different rheological properties Blanc et al. 2011); one thus has to be careful about shear history when characterizing a suspension, to ensure that the same structure is always dealt with. Here we choose to characterize the material as prepared, which we expect to be roughly isotropic given the complexity of the mixing flow. This point is also in favor of the use of a vane-in-cup geometry to study the materials: the use of a vane allows the study of the properties of the prepared material with minimal disturbance of the material structure during the insertion of the tool (Dzuy and Boger 1983).

Finally, following Mahaut et al. (2008a), the procedure is the following:

- a suspension is prepared as detailed is Sec. 3.1 and poured into the cup of the vane-and-cup geometry. The vane tool is then slowly inserted into the material.

- the elastic modulus $G^{\prime}$ is determined through oscillatory shear experiments. As we work with a controlled stress rheometer, a shear stress is imposed rather than a shear strain, in order to get accurately small deformations. The oscillatory stress is imposed during $1 \mathrm{~min}$ at a $2 \mathrm{~Hz}$ frequency. Its amplitude $\tau_{0}$ depends on the sample and is chosen so as to ensure that the strain induced on the tested material is lower than $10^{-3}$ and that all materials are tested in their linear regime. The independence of the results on the choice of $\tau_{0}$ was checked on some materials. We checked the independence of the results on the frequency in the $0.1-10 \mathrm{~Hz}$ range: although the elastic modulus $G^{\prime}(0)$ of the emulsion depends on the frequency, the dimensionless modulus $G^{\prime}(\phi) / G^{\prime}(0)$ does not significantly depend on it; the impact of bubbles is thus properly accounted for. A more detailed study of a possible frequency dependence (which can be expected due to the presence of interfaces) is postponed to another study.

- afterwards, we perform our yield stress measurement with the vane method (Nguyen and Boger 1985; Liddell and Boger 1996): a small rotational velocity, corresponding to a $0.01 \mathrm{~s}^{-1}$ macroscopic shear rate, is imposed on the vane tool during $120 \mathrm{~s}$. We checked that the same effect of the bubbles on the yield stress is observed whatever the low 

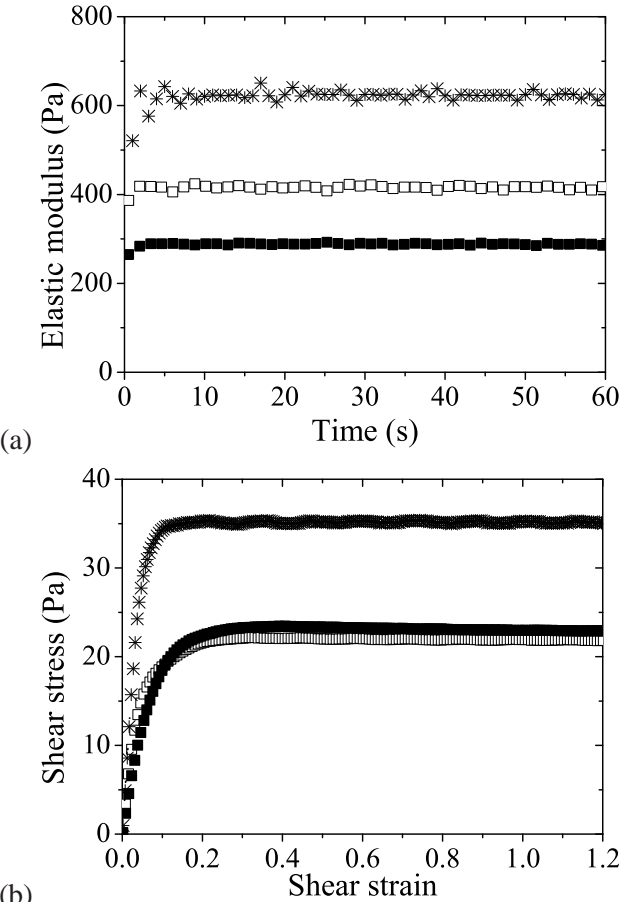

Fig. 2 (a) Elastic modulus G' vs. time after loading when an oscillatory shear stress of amplitude $\tau_{0}=0.35 \mathrm{~Pa}$ is applied at a frequency of $2 \mathrm{~Hz}$ to a pure emulsion (crosses), a suspension of bubbles in the emulsion (filled squares), and in the interstitial emulsion of the suspension (diluted emulsion, empty squares). The bubble volume fraction in the suspension is $43 \%$, the bubble size is $320 \mu \mathrm{m}$. (b) Shear stress vs. shear strain when slowly shearing the same materials from rest at $10^{-2} s^{-1}$.

velocity that is chosen to drive the vane tool. We also checked that the elasticity measurement is nonperturbative: the same yield stress is measured if a zero stress is imposed instead of oscillations before the yield stress measurement

- finally, as the yield stress measurement may induce migration, bubble rising and microstructure anisotropy, any new measurement requires new sample preparation.

Fig. 2 shows raw elastic and yield stress measurements performed in a pure emulsion, in a suspension of $320 \mu \mathrm{m}$ bubbles (made by adding foam to this last emulsion) and in its corresponding interstitial emulsion (made by adding only surfactant solution to the emulsion). $G^{\prime}$ remains constant in time, consistent with the nonthixotropic character of emulsions. This indicates that the only impact of adding surfactant solution and bubbles to the emulsion is a change in the $G^{\prime}$ value: no new significant mechanism of aging has arisen. The elastic modulus $G^{\prime}$ of the material is thus unambiguously defined from this experiment; we study its dependence on the material composition in Sec.4.

On the stress vs. strain plot during the yield stress measurement (Fig. 2b), there is first a linear increase of stress with strain: this corresponds to the elastic deformation of the material. There is then a well-defined plateau, corresponding to the plastic flow at low shear rate (we checked that viscous effects are here negligible), as usually observed in simple yield stress fluids. This plateau defines unambiguously the yield stress $\tau_{y}$ of the materials; its dependence on the material composition is studied in $\mathrm{Sec}$.5

As observed in Fig 2, the uncertainty on the measurement of the elastic modulus and of the yield stress of a given sample is very low (less than $1 \%$ ). The uncertainty on the suspension mechanical properties mostly comes from the reproducibility of the whole sample preparation procedure (and most probably from the dilution of the interstitial emulsion). Since the liquid fraction in the aqueous foam is not controlled, our procedure does not allow us to obtain several samples at the same exact bubble volume fraction. We are thus not able to evaluate rigorously this uncertainty. Nevertheless, from several couples of data obtained in materials prepared at approximately the same volume fraction, we can evaluate the uncertainty on the suspension elastic modulus and yield stress to be of order $5 \%$.

\section{Elastic modulus}

\subsection{Mixtures of foam and emulsion}

In this section, we first study the cases where suspensions of increasing bubble volume fraction are obtained by adding foam to a given emulsion in increasing foam to emulsion mass ratios. We recall that each suspension is compared to the emulsion surrounding the bubbles, made by adding only surfactant solution to the initial emulsion (see Sec.3.1, Fig.11.

In Fig. 3a we plot the values of the elastic modulus $G^{\prime}$ measured in suspensions of $320 \mu \mathrm{m}$ bubbles and in their corresponding interstitial emulsion, as a function of the bubble volume fraction $\phi$ in the suspension. As expected from the dilution of the material by the surfactant solution, the more foam we incorporate (i.e., as $\phi$ increases), the lower the elastic modulus of the interstitial fluid gets. In concentrated emulsions, $G^{\prime}$ is actually known to be a monotonic increasing function of the droplet volume fraction above the jamming packing fraction $\phi_{m}$ (Mason et al. 1995) and to tend to zero at $\phi_{m}$. The inset of Fig. 3 shows the elastic modulus of the interstitial emulsion replotted as a function of the droplet volume fraction $\phi_{d}$ in the emulsion (higher quantities of foam added to the system, and thus higher bubble volume fraction $\phi$ in the suspension, corresponds to lower values of $\phi_{d}$ ); the observed behavior is consistent with the law proposed by Mason et al. (1995).

The elastic moduli of the bubble suspensions are all significantly lower than those of the interstitial emulsions. This means that the impact of adding foam is twofold: (i) there is a decrease of the elastic modulus of the interstitial fluid due to its dilution, and (ii) there is an additional decrease of the 

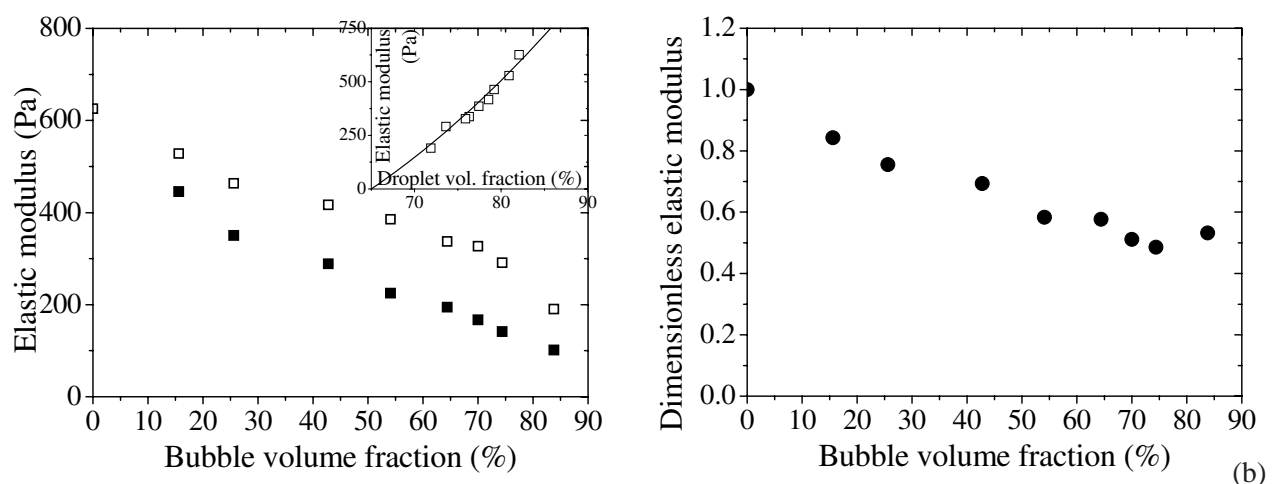

Fig. 3 (a) Elastic modulus vs. bubble volume fraction for the suspensions of bubbles in the emulsion (filled squares), and for the emulsions surrounding the bubbles in the suspensions (diluted emulsions, empty squares). (b) Dimensionless elastic modulus $g(\phi)$ vs. bubble volume fraction $\phi$. The bubble size is $320 \mu \mathrm{m}$. It is recalled that an increasing $\phi$ corresponds to an increasing quantity of foam added to the emulsion, and to a lower droplet volume fraction in the interstitial emulsion. Inset: elastic modulus $G_{i}^{\prime}$ of the interstitial emulsion vs. oil droplet volume fraction $\phi_{d}$ in the emulsion. The line is a fit to the $\phi_{d}\left(\phi_{d}-\phi_{m}\right)$ scaling proposed by Mason et al. (1995) with $\phi_{m}=65 \%$.

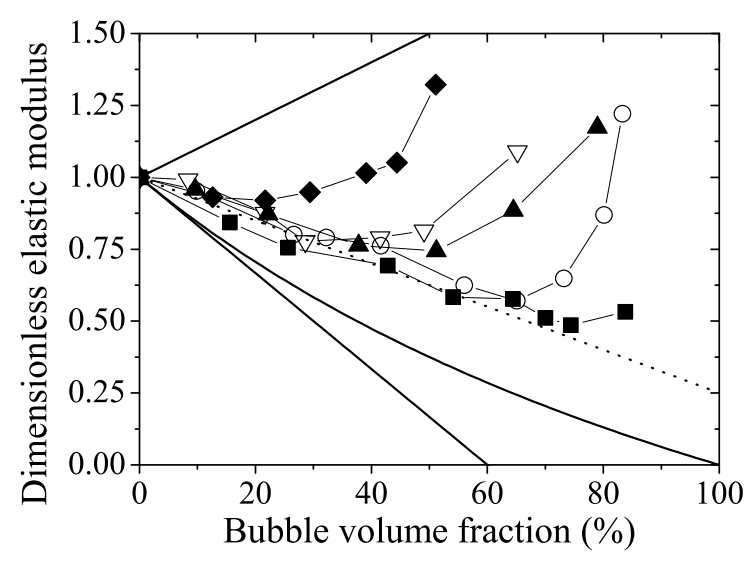

Fig. 4 Dimensionless elastic modulus $g(\phi)$ vs. bubble volume fraction $\phi$, for suspensions of bubble diameter $d=320 \mu \mathrm{m}$ (squares), $260 \mu \mathrm{m}$ (empty circles), $230 \mu \mathrm{m}$ (up triangles), $210 \mu \mathrm{m}$ (empty down triangles), and $110 \mu \mathrm{m}$ (diamonds). The dotted line is a $g(\phi)=1-0.75 * \phi$ equation. The full lines correspond to, from bottom to top: the theoretical value of $g(\phi)$ for suspensions of fully deformable bubbles in the dilute limit (Eq. 9), the theoretical upper bound for isotropic suspensions of fully deformable bubbles (Eq. 10], and the theoretical value of $g(\phi)$ for suspensions of nondeformable bubbles in the dilute limit (Eq. 8).

modulus of the suspension due to the addition of bubbles. To quantify the decrease due solely to the presence of bubbles, we plot the dimensionless elastic modulus $g(\phi)=G^{\prime}(\phi) / G_{i}^{\prime}$ vs. bubble volume fraction $\phi$ in Fig. $3 \mathrm{~b}$; here, $G_{i}^{\prime}$ is the elastic modulus of the interstitial emulsion corresponding to each suspension, i.e., accounting for dilution effects. In this system (suspension of $320 \mu \mathrm{m}$ bubbles), $g(\phi)$ is found to decrease regularly (basically linearly) when $\phi$ increases. E.g., for $75 \%$ of bubbles, $G^{\prime}$ is decreased by a factor 2 due to the presence of bubbles.

In Fig. 4, we now plot all the dimensionless moduli $g(\phi)$ determined in the suspensions of bubbles of diameter $d$ varying between 110 and $320 \mu \mathrm{m}$. Two behaviors are observed. (i) For a volume fraction $\phi$ lower than a critical value $\phi_{c}(d)$ that depends on $d, g(\phi)$ decreases with increasing $\phi$, and, at first glance, all the data seem to collapse onto a roughly linear curve. (ii) Above $\phi_{c}(d), g(\phi)$ is found to increase with $\phi$, and the way it increases depends strongly on $d$. At the highest volume fraction reached in all systems, the elastic modulus of the bubble suspension is up to $35 \%$ higher than that of the interstitial emulsion.

\subsection{Role of bubble deformability}

Let us now try to understand this behavior. As explained in $\mathrm{Sec} 2$ a decrease of the elastic modulus when the bubble volume fraction is increased is the signature of bubble deformability under shear. The fact that $G^{\prime}$ changes from decreasing to increasing with $\phi$ around a critical value $\phi_{c}(d)$ would then mean that bubbles are changed from deformable to nondeformable when the bubble volume fraction is increased. This is a priori surprising as the ability of the bubbles to be deformed under shear should not depend on $\phi$ (as long as the material is not in a 'foam' regime). However, it should be recalled that, with the procedure used here to prepare the material, increasing values of $\phi$ imply decreasing values of the interstitial material elastic modulus $G_{i}^{\prime}$ (Fig. 33). This suggests that the transition is driven by the elastic capillary number $C a_{G}=G_{i}^{\prime} /\left(2 \sigma_{t} / d\right)$, which compares the material and bubble stiffnesses (Sec. 2.2).

To go one step further, we thus plot the $C a_{G}$ values vs. the bubble volume fraction $\phi$ for all the studied systems (Fig. 5); it is assumed here that the surface tension $\sigma_{t}$ between the gas bubbles and the emulsion takes the value of the air/surfactant solution surface tension. At low $\phi$, we observe that $C a_{G}$ is of order of a few units, consistent with the decrease of $G^{\prime}(\phi)$ with $\phi$ : the bubbles behave as soft inclusions (they are deformable). Below a critical value of 


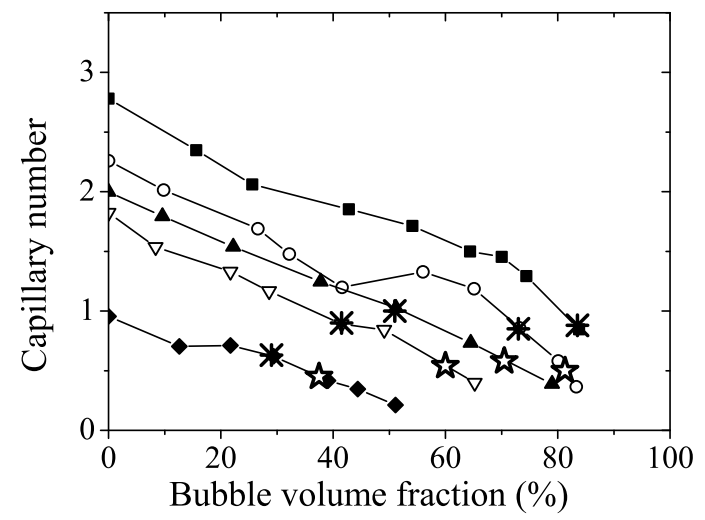

Fig. 5 Elastic capillary number $C a_{G}$ vs. bubble volume fraction $\phi$, for suspensions of bubble diameter $d=320 \mu \mathrm{m}$ (squares), $260 \mu \mathrm{m}$ (empty circles), $230 \mu \mathrm{m}$ (up triangles), $210 \mu \mathrm{m}$ (empty down triangles), and $110 \mu \mathrm{m}$ (diamonds), in the experiments of Fig. 4 The empty stars are $C a_{G}(\phi)$ values corresponding to $g(\phi)=1$ (interpolated from Fig. 4), i.e., to the transition from soft to stiff bubbles. The crosses are $\mathrm{Ca}_{G}(\phi)$ values below which $g(\phi)$ data do not collapse anymore onto a single curve in Fig. 4

$C a_{G}$ that is expected to be of order unity, bubbles should progressively behave as rigid inclusions; from Fig. 5, this should happen above a volume fraction $\phi_{c}^{\prime}(d)$ that depends on $d$, which may explain a the $d$-dependent rise of $G^{\prime}(\phi)$ at high $\phi$. To show that this mechanism may account for our results, we plot in Fig. 5 the $C a_{G}(\phi)$ values corresponding to $g(\phi)=1$, i.e., to the transition from soft to stiff bubbles; these values are interpolated from Fig. 4 A constant critical capillary number $\simeq 0.5$ seems to characterize this transition; we will come back onto this point below. In Fig. 5, we also plot the $C a_{G}(\phi)$ values below which $g(\phi)$ data do not collapse anymore onto a single curve in Fig. 4. Again, a roughly constant capillary number seems to characterize a change in the elastic behavior; here, it likely marks the transition to a regime where the behavior strongly depends on $C a_{G}$. Note that this approach seems to work at bubble volume fraction $\phi$ as high as $80 \%$, although the elastic capillary number is not expected to play exactly the same role in the 'foam' regime, for $\phi$ higher than $\simeq 64 \%$, as in the 'suspension' regime. Things can indeed be more complex in a 'foamy yield stress fluid', as bubbles have to be deformed for purely geometrical reasons.

In the regime where bubble deformability is likely to be important, the dimensionless elastic modulus $g(\phi)$ does not follow the theoretical law for suspensions of fully deformable bubbles in the dilute limit (Eq.9): a $-5 / 3$ slope is indeed expected at low $\phi$ whereas a slope of order of $-3 / 4$ is observed (Fig. 4). Moreover the data fall above the theoretical upper bound of Mori-Tanaka (Eq.10), expected to be valid for bubbles with no surface tension. This indicates that we are not in the limit where bubbles are fully deformable and that elasticity must be stored in the interfaces due to sur- face tension. This naturally leads to an increased rigidity of the system as compared to the case with no surface tension; a theoretical upper bound for this case still has to be computed. This observation is consistent with the fact that the elastic capillary number $C a_{G}$ is only of the order of a few units in all the studied systems (Fig. 57).

At the lowest $C a_{G}$ values, although the bubbles are expected to start behaving as stiff inclusions, the dimensionless elastic modulus $g(\phi)$ is well below the theoretical law for suspensions of nondeformable bubbles in the dilute limit (Eq. 8). This would mean that the bubbles are far from their nondeformability limit, consistent with the fact that $C a_{G}$ is only slightly less than unity in these cases.

\subsection{Suspensions of bubbles at fixed capillary number}

To better show that the elastic behavior is controlled by $\mathrm{Ca}$ and to quantify the impact of a change in $C a_{G}$ on the value of $g(\phi)$, we now study suspensions of bubbles prepared with the same interstitial emulsion (of elastic modulus $G_{0}^{\prime}=285 \mathrm{~Pa}$ ) between the bubbles at any $d$ and $\phi$ (see Sec. 3, Fig. 1b). For a given bubble size, the $g(\phi)$ curve is then characteristic of a given value of $C a_{G}$. We study suspensions made of bubbles of three different diameters: $100 \mu \mathrm{m}, 300 \mu \mathrm{m}$, and $1.6 \mathrm{~mm}$; they are characterized by capillary numbers $C a_{G}$ equals respectively to $0.4,1.2$, and 6.3 . Only data for $\phi<60 \%$ are shown: we do not study foams.

In Fig. 6, we observe that the behavior depends significantly on $C a_{G}$. In the three studied systems, $g(\phi)$ decreases with $\phi$, and decreases more and more with $\phi$ as $C a_{G}$ is increased 3 . A linear fit to the data (with $g(0)=1$ ) yields slopes equal to $-0.17,-0.72$, and -1.1 for increasing values of $C a_{G}$; we recall that, for fully deformable bubbles, the slope is expected to be equal to -1.66 in the dilute limit.

The behavior at the lowest value of $C a_{G}$ investigated here (0.4) is close to a $g(\phi) \simeq 1$ curve. This confirms the observations on Fig 4 , where $g(\phi) \simeq 1$ was obtained for $C a_{G} \simeq$ 0.5 at different bubble diameters (see Fig. 5). In this case, the bubbles then seem to behave as equivalent elastic spheres of elastic modulus equal to that of the interstitial material; this may mean that in general bubbles behave as equivalent elastic particles of modulus $G_{e q}^{\prime}$ equal to the capillary pressure $4 \sigma_{t} / d$. At the highest value of $C a_{G}$ investigated, the $g(\phi)$ curve falls slightly above the theoretical upper bound for bubbles with no surface tension (Eq. 10). This suggests that the bubbles do not behave yet as fully deformable bubbles, but they may be close to this asymptotic limit.

\footnotetext{
3 Since the elastic moduli of the three systems are measured in the linear regime where their behavior does not depend on the strain amplitude, we also note that different $g(\phi)$ values can be obtained for a same value of $C a_{\tau}$ (see Eq. 13, this shows that $C a_{\tau}$ is not a relevant parameter in this regime.
} 


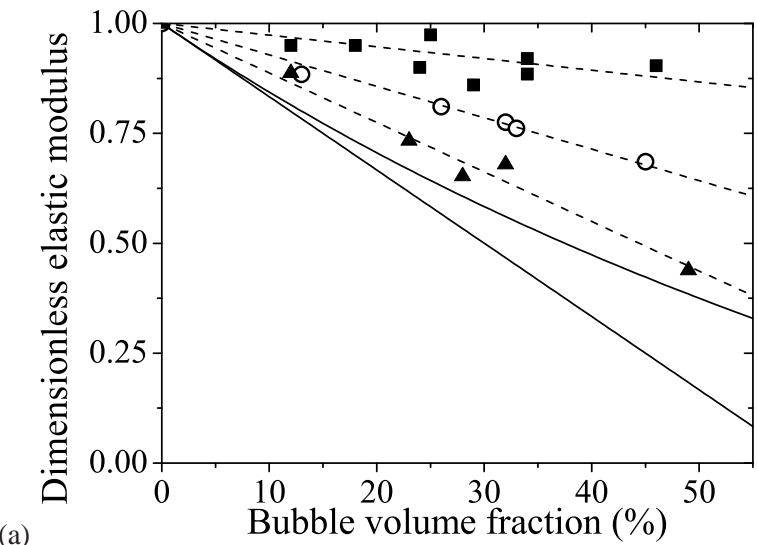

(a)

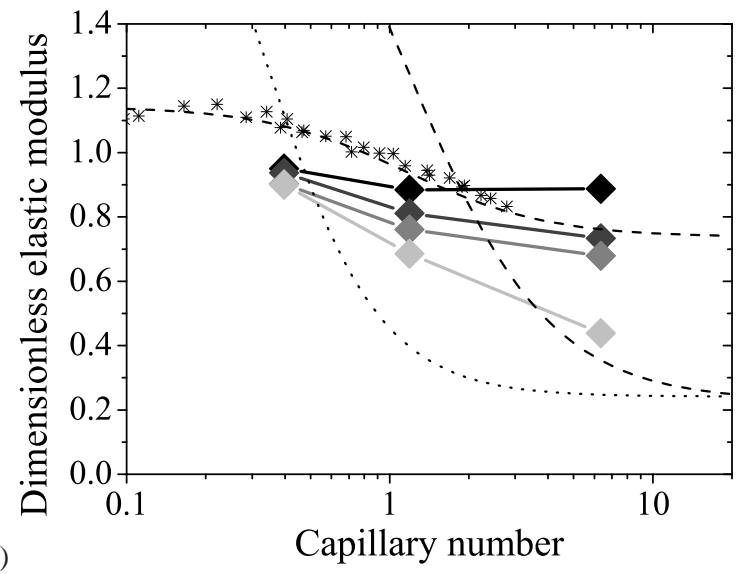

Fig. 6 (a) Dimensionless elastic modulus $g(\phi)=G^{\prime}(\phi) / G^{\prime}(0)$ vs. bubble volume fraction $\phi$, for suspensions of bubble diameter $d=100 \mu \mathrm{m}$ (squares), $300 \mu \mathrm{m}$ (empty circles), and $1.6 \mathrm{~mm}$ (triangles), in an emulsion of elastic modulus $G^{\prime}(0)=285 \mathrm{~Pa}$. The full lines correspond to, from bottom to top: the theoretical value of $g(\phi)$ for suspensions of fully deformable bubbles in the dilute limit (Eq. 9) and the theoretical upper bound for isotropic suspensions of fully deformable bubbles (Eq. 10); the dotted lines are linear fits to the data (their slopes are, from top to bottom: $-0.17,-0.72,-1.1$ ). (b) Dimensionless elastic modulus $g(\phi)$ vs. elastic capillary number $C a_{G}$ at different volume fraction (diamonds): $\phi \simeq 11-12 \%$ (black), $\phi \simeq 23-26 \%$ (dark grey), $\phi \simeq 32-34 \%$ (medium grey), $\phi \simeq 45-49 \%$ (light grey); crosses are dimensionless viscosities measured in suspensions of bubbles in a Newtonian fluid vs. viscous capillary number $C a_{\eta}$ at $\phi=11.5 \%$ (data replotted from Rust and Manga (2002a)); the dashed lines are the phenomenological expression proposed by Rust and Manga (2002a) (Eq. 9 of their paper) for $\phi=11.5 \%$ and $\phi=47.5 \%$; the dotted line is the phenomenological expression proposed by $\mathrm{Pal}$ (2004) (Eqs. 12 and 35-38 of the paper) for $\phi=47.5 \%$.

In Fig. 6b, we now plot the dimensionless elastic modulus $g(\phi)$ as a function of the capillary number $C a_{G}$ for different constant bubble volume fractions $\phi$. Given the limited amount of data, and the difficulty to target the exact same value of $\phi$ in different systems, this plot is rather qualitative; data are indeed plotted for $\phi$ values in four different intervals: between 11 and $12 \%$, between 23 and $26 \%$, between 32 and $34 \%$, and between 45 and $49 \%$. It is observed that $g(\phi)$ varies smoothly with $C a_{G}$. This evolution can be compared to that observed by Rust and Manga (2002a) for suspensions of bubbles in Newtonian fluids: the dimensionless viscosities they have measured at $\phi=11.5 \%$ are plotted vs $C a_{\eta}$ in Fig. 6 , together with the empirical function they have proposed to account for their data and for other data of the literature; the equation proposed by $\mathrm{Pal}$ (2004) is also shown. The Rust and Manga (2002a) data decrease more rapidly with $C a_{\eta}$ than ours do with $C a_{G}$ for the same value of $\phi$, and the $g(\phi)$ evolution predicted by both the Rust and Manga (2002a) and the Pal (2004) empirical equations at high $\phi$ is much more abrupt than that we observe. Note however that several issues prevent from an in-depth comparison. (i) Rust and Manga (2002a) data were obtained on polydisperse systems, and only up to $\phi=16 \%$. (ii) We are not aware of other data obtained at intermediate $C a_{c}$ values in the literature; at high $\phi$, the Rust and Manga (2002a) and Pal (2004) equations have been fitted only to the asymptotic viscosities corresponding to nondeformable and fully deformable bubbles. It thus seems that the behavior of concentrated suspensions of bubbles in a Newtonian fluid at capillary number of order unity is not yet known. (iii) The two studied problems are not exactly the same: in steady-state flows, bubbles are deformed by shear and reach a steady shape. Here, the bubbles are only slightly deformed by the low amplitude oscillatory measurement: although they behave as soft deformable particles, they remain basically spherical. Further theoretical work is needed to better understand this difference.

The smooth variation of the dimensionless modulus with $C a_{G}$ observed in Fig. 6b explains the apparent data collapse in the first series of experiments (Fig. 4): although data corresponded to different capillary numbers (Fig.55), those were ranging only between 1 and 2.5 ; consistently, the -0.75 slope observed for the line onto which data collapse in Fig. 6b is close to the slope here observed for a constant $C a_{G}=1.2$.

Note that it was not possible to study systems of lower $C a_{G}$ value. Lower values of the bubble size would indeed pose finite size effects problems as scale separation between bubbles/droplets would not be ensured anymore. Emulsions of lower elastic modulus should have a higher droplet size, which poses again finite size effect problems, or a lower droplet volume fraction, which poses the problem of reproducibility of the systems preparation (the emulsion elastic modulus is highly sensitive to a change of the droplet volume fraction at the approach of its jamming packing fraction). In future works, a possible way to prepare materials at lower $C a_{G}$ may be to use surfactants that would lower the oil/water interfacial tension significantly more than the air/water surface tension. It was also not possible to study systems of higher $C a_{G}$ value because bubbles tends to be broken by the mixing process in such systems (see next section). In the future, it will be necessary to use a new prepara- 
tion method to disperse homogeneously bubbles in an emulsion without mixing.

\section{Yield stress}

\subsection{Mixtures of foam and emulsion}

As in Sec. 4, we first study the cases in which suspensions of increasing bubble volume fraction are obtained by adding foam to a given emulsion in increasing foam to emulsion mass ratios.

Fig. $7 \mathrm{~h}$ shows the yield stress values $\tau_{y}$ measured in suspensions of $320 \mu \mathrm{m}$ bubbles and in their corresponding interstitial emulsion, as a function of the bubble volume fraction $\phi$ in the suspension. Because of its dilution by the surfactant solution brought in by the foam, as already observed for the elastic modulus, the yield stress of the interstitial fluid decreases when the quantity of foam incorporated in the emulsion is increased (i.e., when $\phi$ increases). The inset of Fig. 7h shows the yield stress of the interstitial emulsion replotted as a function of the droplet volume fraction in the emulsion; our data are consistent with the observations of Mason et al. (1996) and with the empirical law they propose to model the yield stress of concentrated emulsions.

The yield stress of the bubble suspensions is found to decrease with $\phi$ similarly to that of the interstitial emulsion. This would mean that, as regards its plastic properties, the main impact of adding a foam to the material is to dilute the interstitial fluid. The impact of the presence of bubbles can be more precisely evaluated by plotting the suspension dimensionless yield stress $\tau_{y}(\phi) / \tau_{y, i}$ vs. bubble volume fraction $\phi$ (Fig. 7b); here, $\tau_{y, i}$ is the yield stress of the interstitial emulsion corresponding to each suspension, i.e., accounting for dilution effects. In this system (suspension of $320 \mu \mathrm{m}$ bubbles), up to $\phi=80 \%, \tau_{y}(\phi) / \tau_{y, i}$ is found to remain basically constant and equal to 1 . A significant increase of $\tau_{y}(\phi) / \tau_{y, i}$ is observed only at the highest volume fraction $(85 \%)$.

In Fig. 8, we plot the dimensionless yield stresses determined for all our suspensions, of bubble diameter $d$ varying between 110 and $320 \mu \mathrm{m}$. Two behaviors are observed. (i) For volume fractions $\phi$ lower than a critical value $\phi_{c}^{\prime}(d)$ that depends on $d$, all the data seem to collapse onto a single line: $\tau_{y}(\phi) / \tau_{y, i}$ is basically constant and equal to 1 , i.e., the yield stress of the suspensions is equal to that of their interstitial emulsion. (ii) Above $\phi_{c}^{\prime}(d), \tau_{y}(\phi) / \tau_{y, i}$ is found to increase with $\phi$, in a way that depends on $d$. In this last regime, the yield stress of the suspension can be up to 3.7 times higher than that of the interstitial emulsion. In the following, we first focus on the first regime.

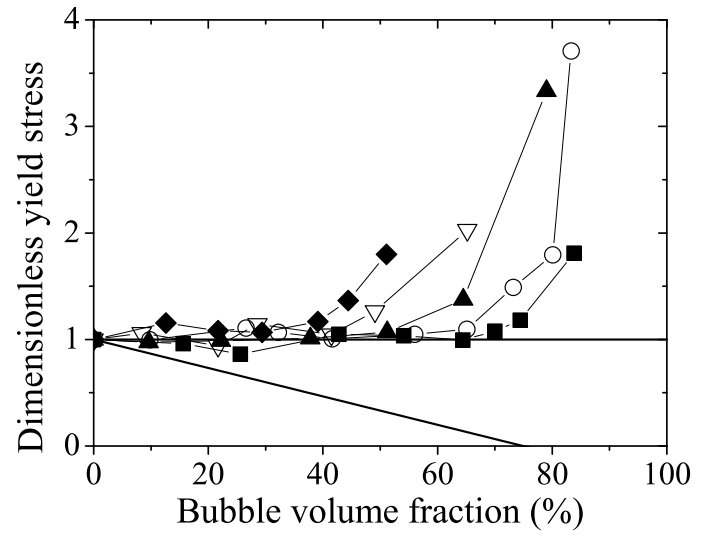

Fig. 8 Dimensionless yield stress $\tau_{y}(\phi) / \tau_{y, i}$ vs. bubble volume fraction $\phi$, for suspensions of bubble diameter $d=320 \mu \mathrm{m}$ (squares), $260 \mu \mathrm{m}$ (empty circles), $230 \mu \mathrm{m}$ (up triangles), $210 \mu \mathrm{m}$ (empty down triangles), and $110 \mu \mathrm{m}$ (diamonds). The full lines correspond to, from bottom to top: the theoretical value of $\tau_{y}(\phi) / \tau_{y, i}$ for suspensions of fully deformable bubbles in the dilute limit (Eq. [15, and the theoretical value of $\tau_{y}(\phi) / \tau_{y, i}$ for suspensions of nondeformable bubbles in the dilute limit (Eq. 14).

\subsection{Role of deformability}

As shown in Sec 2, a value of $\tau_{y}(\phi) / \tau_{y}(0) \approx 1$ is expected for nondeformable bubbles in a yield stress fluid (Eq. 14), whereas a strong decrease (with a slope -4/3) should be observed for deformable bubbles (Eq. 15). Although the elastic modulus of these suspensions decreases with $\phi$, this analysis suggests that, for $\phi<\phi_{c}^{\prime}(d)$, bubbles are not deformed at yield. This is fully consistent with the values of the yield capillary number $C a_{\tau_{y}}=\tau_{y} /\left(2 \sigma_{t} / d\right)$ of the studied materials, which are all small - of order 0.01 to 0.1 . It thus seems that the elastic and plastic behaviors of the bubble suspensions are governed by two independent capillary numbers.

To confirm that the behavior is controlled by $C a_{\tau_{y}}$, we now study suspensions of bubbles prepared with a same interstitial emulsion surrounding the bubbles at any $\phi$ (see Sec. 3). In Fig. 9 we first present the dimensionless yield stresses measured in suspensions of $100 \mu \mathrm{m}, 300 \mu \mathrm{m}$ and $1.6 \mathrm{~mm}$ bubbles in a $9 \mathrm{~Pa}$ yield stress emulsion; the corresponding plastic capillary numbers are 0.01, 0.04, and 0.2. We observe that all data are consistent with $\tau_{y}(\phi) / \tau_{y}(0)=1$, meaning that up to $C a_{\tau_{y}} \simeq 0.2$, bubbles are not deformed by shear.

To go one step further and to investigate the possibility of a regime where bubbles are deformed by shear, we need to increase the value of $C a_{\tau_{y}}$ as much as possible, which can be obtained by increasing the material yield stress and the bubble diameter. We have prepared materials with the goal of studying suspensions of $3 \mathrm{~mm}$ bubbles in emulsions of

4 We remind that this should be strictly true in the dilute limit only: we do not yet have a prediction for all values of $\phi$. 

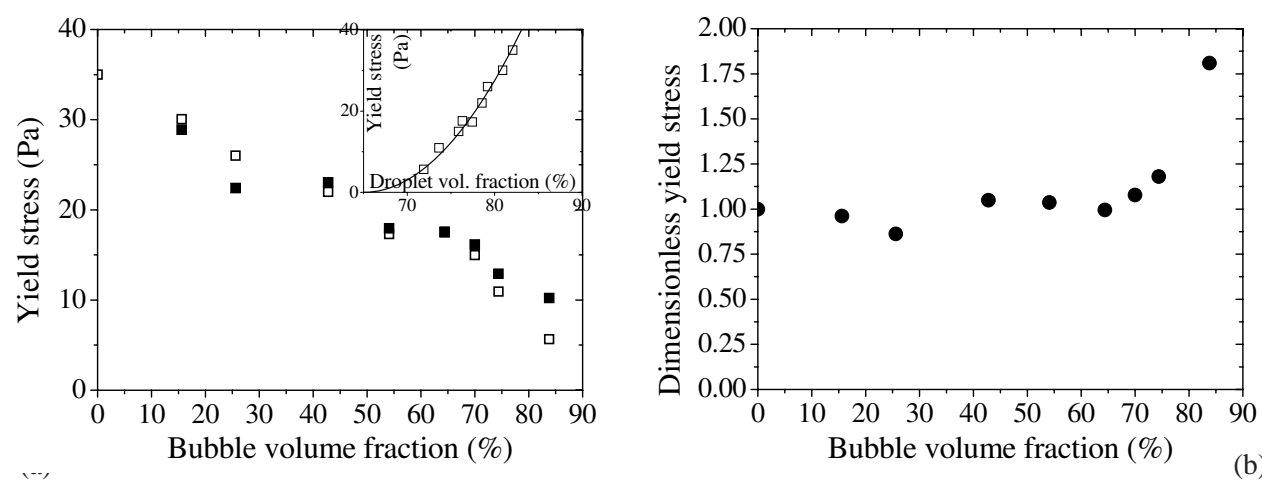

Fig. 7 (a) Yield stress vs. bubble volume fraction for the suspensions of bubbles in the emulsion (filled squares), and for the emulsions surrounding the bubbles in the suspensions (diluted emulsions, empty squares). (b) Dimensionless yield stress $\tau_{y}(\phi) / \tau_{y, i}$ vs. bubble volume fraction $\phi$. The bubble size is $320 \mu \mathrm{m}$. It is recalled that increasing $\phi$ corresponds to an increasing quantity of foam added to the emulsion, and to a lower droplet volume fraction in the interstitial emulsion. Inset: yield stress $\tau_{v, i}$ of the interstitial emulsion vs. oil droplet volume fraction $\phi_{d}$ in the emulsion. The line is a fit to the $\left(\phi_{d}-\phi_{m}\right)^{2}$ scaling proposed by Mason et al. (1996) with $\phi_{m}=65 \%$.

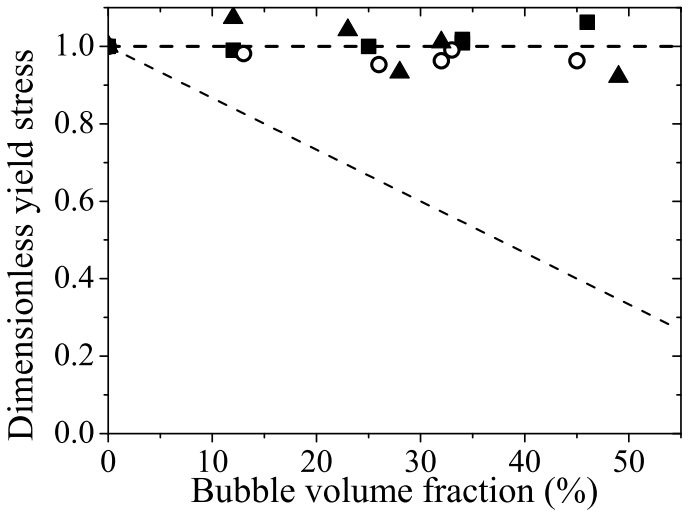

Fig. 9 Dimensionless yield stress $\tau_{y}(\phi) / \tau_{y}(0)$ vs. bubble volume fraction $\phi$, for suspensions of bubble diameter $d=100 \mu \mathrm{m}$ (squares), $300 \mu \mathrm{m}$ (empty circles), and $1.6 \mathrm{~mm}$ (triangles), in an emulsion of yield stress $\tau_{y}(0)=9 \mathrm{~Pa}$. The dashed lines correspond to, from bottom to top: the theoretical value of $\tau_{y}(\phi) / \tau_{y}(0)$ for suspensions of fully deformable bubbles in the dilute limit (Eq. [15), and the theoretical value of $\tau_{y}(\phi) / \tau_{y}(0)$ for suspensions of nondeformable bubbles in the dilute limit (Eq.14).

9, 19, and $72 \mathrm{~Pa}$ yield stress (corresponding to target plastic capillary numbers of order $0.4,0.8$, and 3 ). In this end, foams of $3 \mathrm{~mm}$ bubbles were mixed with emulsions of initial yield stress (before mixing) equal to 19 and $87 \mathrm{~Pa}$ (corresponding to initial capillary numbers of order 0.8 and 3.6). Photographs of the aqueous foam and of the prepared bubble suspensions are shown in Fig. 10, photograph of a suspension of $1.6 \mathrm{~mm}$ bubbles is also presented for comparison.

In the case of the suspension prepared with an aqueous foam of $1.6 \mathrm{~mm}$ diameter bubbles, it is observed that the bubbles in the suspension have the same size as in the aqueous foam. By contrast, all suspensions prepared with an aqueous foam of $3 \mathrm{~mm}$ diameter bubbles are polydisperse: small bubbles have appeared, due to shear-induced bubble rupture during the mixing process (we checked that the air content in the suspension matches that brought in by the foam into the emulsion). Moreover, the bubbles are smaller for higher values of the initial emulsion yield stress and of the final interstitial emulsion yield stress. This is consistent with a mechanism of shear-induced bubble breakup, which is expected to depend on the ability of the bubbles to be deformed by shear. As for bubble and droplet breakup in Newtonian fluids (Larson 1999), we expect this property to be governed by a capillary number, the relevant one being here the plastic capillary number $C a_{\tau} 5$. Consistently, in the experiments of Fig. 10, the initial values of the plastic capillary number leading to breakup are of order 0.8 to 3.6. It is worth noting that the final capillary numbers of the prepared suspensions, based on the smallest bubble size observable on the pictures, is of order 0.2 in the 3 systems. Note also, that the final dimensionless yield stress of the first 2 systems is of order 1 (we could not get a reliable measurement of this property in the third system, which was inhomogeneous after mixing). It thus appears that (i) for $C a_{\tau_{y}}$ values of order 0.2 or less, the bubbles behave as nondeformable inclusions under shear and that the suspension yield stress is equal to that of the interstitial emulsion, and that (ii) for initial values of $C a_{\tau_{y}}$ above a critical plastic capillary number of order 0.2 , bubbles are deformed and broken in the sheared suspension. This might imply that, in any bubble suspension prepared by mixing a foam and a paste, the final bubble size in the system is always such that $C a_{\tau_{y}} \lesssim 0.2$, and that its yield stress is equal to that of the interstitial paste. This possible important feature has to be studied in more detail with well-controlled mixing procedures.

\footnotetext{
${ }^{5}$ If mixing is rapid and if viscous effects are important, for a constitutive behavior of the form $\tau=\tau_{y}+f(\dot{\gamma})$, the relevant number might
} rather be $\frac{\tau_{y}+f(\dot{\gamma})}{2 \sigma_{t} / d}$. 


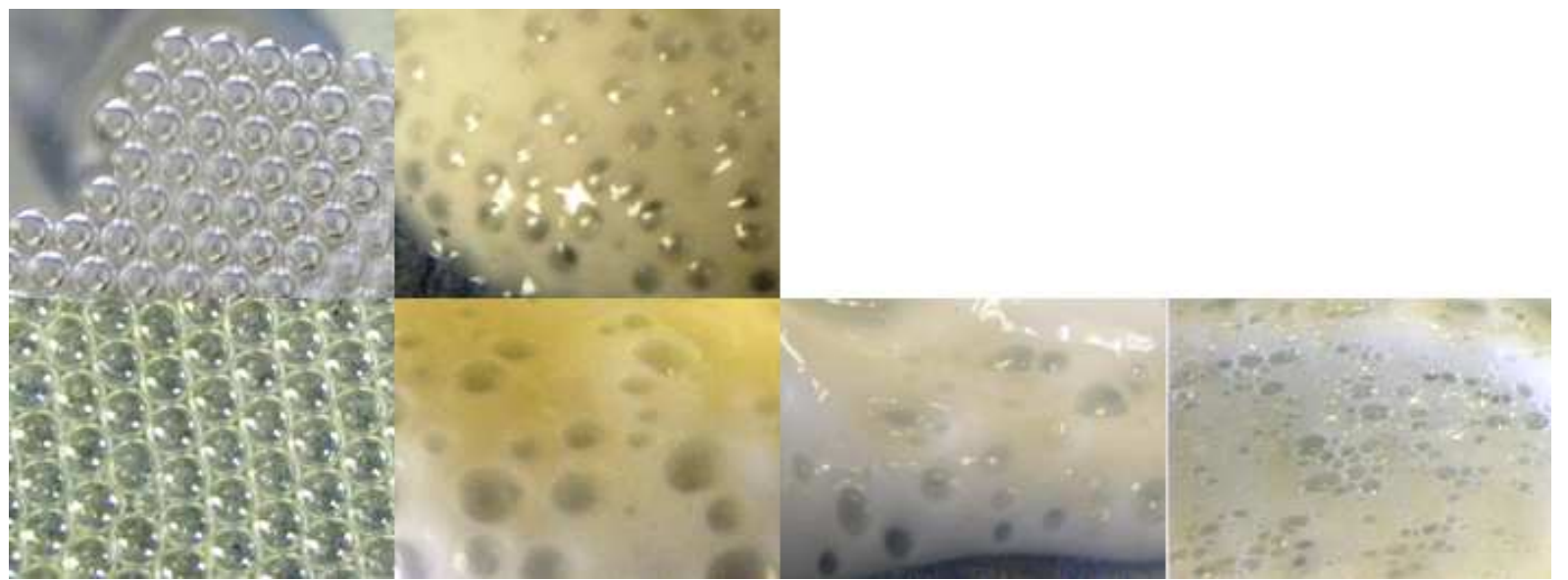

Fig. 10 Top: Pictures of an aqueous foam made of $1.6 \mathrm{~mm}$ diameter bubbles (left) and of a suspension of $1.6 \mathrm{~mm}$ bubbles made by mixing the foam with a $19 \mathrm{~Pa}$ yield stress emulsion (final interstitial emulsion yield stress: $9 \mathrm{~Pa}$ ) [same scale for both images]. Bottom: Pictures of an aqueous foam made of $3 \mathrm{~mm}$ diameter bubbles (left) and of a suspension of $3 \mathrm{~mm}$ bubbles made by mixing the foam with (from left to right): a $19 \mathrm{~Pa}$ yield stress emulsion (final interstitial emulsion yield stress: 9 Pa), a 87 Pa yield stress emulsion (final interstitial emulsion yield stress: 19 Pa), and a $87 \mathrm{~Pa}$ yield stress emulsion (final interstitial emulsion yield stress: $72 \mathrm{~Pa}$ ) [same scale for the 4 images].

5.3 Behavior at high $\phi$ : 'foam' regime and role of finite size effects

We finally come back onto the abrupt increase of the bubble suspension yield stress observed at high volume fraction (Fig. 4). An increase of $\tau_{y}$ with $\phi$ above a $d$-dependent critical value $\phi_{c}^{\prime}(d)$ is a priori surprising as all our results are consistent with bubbles behaving as rigid inclusions at low and moderate $\phi$ values, the plastic capillary number being small; the decrease of $C a_{\tau_{y}}$ with $\phi$ due to the interstitial emulsion dilution is thus not expected to play any role here, in contrast with what was observed with the elastic modulus. Another mechanism should thus be proposed.

At high volume fraction, bubbles have to be deformed for geometric reasons as in aqueous foams. This regime should typically occur for $\phi>\phi_{f}$ with $\phi_{f} \approx 64 \%$ if films are allowed to be as thin as possible. An important characteristic of the studied system is that film thickness is here limited by the microstructure of the emulsion: film thinning stops when the oil droplets cannot be expelled anymore, which occurs when the film thickness is of the order of a few droplet diameters (Goyon et al. 2010). It is expected that flow of the confined emulsion is harder to enforce than that of the bulk material, due to its lack of disorder; this would explain the increase in the suspension yield stress when reaching this 'foam' regime. By contrast, this should not affect the elastic properties of the material. As the minimum distance between bubbles is fixed by the oil droplet size, the maximum packing fraction $\phi_{f}(d)$ delimiting the 'suspension' and 'foam' regimes should depend on the bubble diameter $d$. Noting $e$ the minimum film thickness and $d_{d}$ the oil droplet diameter, and assuming $\phi_{f} \approx 64 \%$ when $d>>d_{d}, \phi_{f}(d)$ can actually be evaluated as $\phi_{f}(d)=0.64 /(1+e / d)^{3}$. Assum- ing a minimum film thickness $e \approx 4 d_{d}$ (Goyon et al. 2010), this yields $\phi_{f}$ values ranging between $42 \%$ for the $110 \mu \mathrm{m}$ bubbles and $55 \%$ for the $320 \mu \mathrm{m}$ bubbles. Note that the proposed mechanism is probably not specific to suspensions of bubbles in emulsions: the same might occur with any paste since their microstructure is often of the order of $1 \mu \mathrm{m}$.

This minimum allowable film thickness has another important consequence, as pointed out by Goyon et al. (2010): bubble suspensions cannot exist at volume fractions higher than a $d$-dependent value $\phi_{m}(d)$ that is fixed by the minimum film thickness $e$. This is the 'dry' limit of 'foamy yield stress fluids'. Assuming a structure of Kelvin cells in this limit, Goyon et al. (2010) have evaluated $\phi_{m} \approx 1-1 /(1+$ $0.3 d / e)$; this would yield $\phi_{m}$ values ranging between $66 \%$ for the $110 \mu \mathrm{m}$ bubbles and $85 \%$ for the $320 \mu \mathrm{m}$ bubbles. This might explain why could not explore the same ranges of volume fractions for different bubble sizes in our experiments (see Figs. 4 and 8): we did not manage to study suspensions of $110 \mu \mathrm{m}$ bubbles at volume fractions higher than $\simeq 55 \%$, whereas suspensions of $320 \mu \mathrm{m}$ bubbles were studied up to $\simeq 85 \%$.

\section{Conclusion}

We have studied the rheological behavior of mixtures of foams and pastes. We have designed well-defined systems by mixing monodisperse foams and monodisperse concentrated emulsions, characterized by large bubble to oil droplet size ratios. These materials are model suspensions of bubbles in a yield stress fluid. We have shown that the elastic and plastic behaviors of these materials are governed by two different dimensionless numbers: the elastic capillary num- 
ber $C a_{G}$, which is the ratio of the paste elastic modulus to the bubble capillary pressure, and the plastic capillary number $C a_{\tau_{y}}$, which is the ratio of the paste yield stress to the bubble capillary pressure.

In our systems, $C a_{G}$ ranges from 0.3 to 10 and the dimensionless elastic modulus of the material decreases with the bubble volume fraction: bubbles behave as soft elastic inclusions. This decrease is all the sharper as $C a_{G}$ is high, which accounts for the softening of the bubbles as compared to the paste. The transition from soft to stiff bubbles seems to occur at $C a_{G} \simeq 0.4$. When mixing a foam and a paste, in some conditions, two contradictory effects might thus be observed as $\phi$ is increased: (i) a decrease of the elastic modulus due to the dilution of the interstitial material by the surfactant solution brought in by the foam, and (ii) an increase of the dimensionless elastic modulus due to the consequent decrease of $C a_{G}$, if values lower than $\simeq 0.4$ are reached. Further investigation is needed to cover a wider range of $C a_{G}$ values and in particular to characterize the regime of stiff bubbles. Theoretical developments based on micromechanical approaches are in progress and should allow the modelling of the elastic modulus as a function of the bubble volume fraction and of the elastic capillary number.

For $C a_{\tau_{y}}$ values lower than $\simeq 0.2$, the dimensionless yield stress of the suspensions is found to be constant and equal to 1 in most cases, consistent with our predictions for the nonlinear behavior of suspensions of nondeformable bubbles. We have tried to prepare systems with a target value of $C a_{\tau_{y}}$ larger than 0.2. Bubble breakup is observed during mixing of these systems, and the final bubble size seems to be set by the paste yield stress: it is smaller when the paste yield stress is higher. This preliminary result is of high practical importance: it might imply that, in any bubble suspensions prepared by mixing a foam and a paste, the final bubble size in the system is always such that $C a_{\tau_{y}} \lesssim 0.2$, and that its yield stress is equal to that of the interstitial paste. Shearinduced bubble breakup has to be studied in more detail as a function of shear history, to better understand which suspensions of bubbles are produced by a given mixing process. To perform such studies, new procedures are needed to disperse homogeneously unbroken bubbles of controlled size in a given yield stress fluid at high values of the plastic capillary number.

At high bubble volume fraction $\phi$, we have observed a regime where the yield stress increases abruptly with $\phi$, the transition volume fraction being lower for lower bubble to droplet size ratio. This is understood as a 'foamy yield stress fluid' regime, where the paste mesoscopic constitutive elements (here, the oil droplets) are strongly confined in the films between the bubbles. We are currently conducting further investigations to better characterize and understand this regime, which is of the highest importance for practical issues.
Acknowledgements We thank Mohammed Bouricha for help on some of the experiments.

We acknowledge funding from Saint-Gobain Recherche.

\section{References}

Ancey C, Jorrot H (2001) Yield stress for particle suspensions within a clay dispersion. J Rheol 45:297-319

Baravian C, Lalante A, Parker A (2002) Vane rheometry with a large, finite gap. Appl Rheol 12:81-87

Blanc F, Peters F, Lemaire E (2011) Local transient rheological behavior of concentrated suspensions. J Rheol 55:835-854

Chateau x, Ovarlez G, Luu Trung K (2008) Homogenization approach to the behavior of suspensions of noncolloidal particles in yield stress fluids. J Rheol 52:489-506

Coussot P (2005) Rheometry of Pastes, Suspensions and Granular Materials. John Wiley \& Sons, Hoboken

Coussot P, Tabuteau H, Chateau X, Tocquer L, Ovarlez G (2006) Aging and solid or liquid behavior in pastes. J Rheol 50:975-994

Denkov ND, Tcholakova S, Golemanov K, Ananthpadmanabhan KP, Lips A (2009) The role of surfactant type and bubble surface mobility in foam rheology. Soft Matter 5:3389-3408

Dormieux L, Kondo D, Ulm FJ (2006) Microporomechanics. John Wiley \& Sons, Hoboken

Dubash N, Frigaard IA (2004) Conditions for static bubbles in viscoplastic fluids. Phys Fluids 16:4319-4330

Dubash N, Frigaard IA (2007) Propagation and stopping of air bubbles in Carbopol solutions. J Non-Newtonian Fluid Mech 142:123-134

Dutta A, Chengara A, Nikolov AD, Wasan DT, Chen K, Campbell B (2004a) Texture and stability of aerated food emulsions-effects of buoyancy and Ostwald ripening. J Food Eng 62:169-175

Dutta A, Chengara A, Nikolov AD, Wasan DT, Chen K, Campbell B (2004b) Destabilization of aerated food products: effects of Ostwald ripening and gas diffusion. J Food Eng 62:177-184

Dzuy NQ, Boger DV (1983) Yield stress measurement for concentrated suspensions. J Rheol 27:321

Frankel NA, Acrivos A (1970) The Constitutive Equation for a Dilute Emulsion. J Fluid Mech 44:65-78

Gadala-Maria F, Acrivos A (1980) Shear-induced structure in a concentrated suspension of solid spheres. J Rheol 24:799-814

Gandolfo FG, Rosano HL (1997) Interbubble gas diffusion and the stability of foams. J Colloid Interface Sci 194:31-36

Geiker MA, Brandl M, Thrane L, Nielsen NF (2002a) On the Effect of Coarse Aggregate Fraction and Shape on the Rheological Properties of Self-Compacting Concrete. Cement Concrete Aggr 24:3-6

Geiker MR, Brandl M, Thrane LN, Bager DH, Wallevik O (2002b) The effect of measuring procedure on the apparent rheological properties of self compacting concrete. Cem Concr Res 32:1791-1795

Gonnermann HM, Manga M (2007) The fluid mechanics inside a volcano. Annu Rev Fluid Mech 39:321-356

Goyon J, Bertrand F, Pitois O, Ovarlez G (2010) Shear Induced Drainage in Foamy Yield-Stress Fluids. Phys Rev Lett 104:128301

Griffiths RW (2000) The dynamics of lava flows. Annu Rev Fluid Mech $32: 477-518$

Hashin Z, Shtrikman S (1963) A variational approach to the theory of the elastic behaviour of multiphase materials. J Mech Phys Solids 11:127-140

Keentok M, Milthorpe JF, O’Donovan E (1985) On the shearing zone around rotating vanes in plastic liquids: theory and experiment. J Non-Newtonian Fluid Mech 17:23-35

Koczo K, Lobo LA, Wasan DT (1992) Effect of oil on foam stability: Aqueous foams stabilized by emulsions. J Colloid Interface Sci 150:492-506

Larson RG (1999)The structure and rheology of complex fluids. Oxford University Press, New York 
Ley MT, Folliard KJ, Hover KC (2009) Observations of air-bubbles escaped from fresh cement paste. Cem Concr Res 39:409-416

Leighton D, Acrivos A (1987) The shear-induced migration of particles in concentrated suspensions. J Fluid Mech 181:415-439

Liddell PV, Boger DV (1996) Yield stress measurements with the vane. J Non-Newtonian Fluid Mech 63:235-261

Llewellin EW, Mader HM, Wilson SDR (2002) The rheology of a bubbly liquid. P R Soc A 458:987-1016

Mabille C, Schmitt V, Gorria P, Leal Calderon F, Faye V, Deminière B, Bibette J (2000) Rheological and Shearing Conditions for the Preparation of Monodisperse Emulsions. Langmuir, 16:422-429

Mahaut F, Chateau X, Coussot P, Ovarlez G (2008a) Yield stress and elastic modulus of suspensions of noncolloidal particles in yield stress fluids. J Rheol 52:287-313

Mahaut F, Mokéddem S, Chateau X, Roussel N, Ovarlez G (2008b) Effect of coarse particle volume fraction on the yield stress and thixotropy of cementitious materials. Cem Concr Res 38:12761285

Mason TG, Bibette J, Weitz DA (1995) Elasticity of Compressed Emulsions. Phys Rev Lett 75:2051-2054

Mason TG, Bibette J, Weitz DA (1996) Yielding and Flow of Monodisperse Emulsions. J Colloid Interface Sci 179:439-448

Mewis J, Wagner N (2012) Colloidal suspension rheology. Cambridge University Press, Cambridge

Nguyen QD, Boger DV (1985) Direct yield stress measurement with the vane method. J Rheol 29:335-347

Ovarlez G, Bertrand F, Rodts S (2006) Local determination of the constitutive law of a dense suspension of noncolloidal particles through magnetic resonance imaging. J Rheol 50:259-292

Ovarlez G, Rodts S, Ragouilliaux A, Coussot P, Goyon J, Colin A (2008) Wide-gap Couette flows of dense emulsions: Local concentration measurements, and comparison between macroscopic and local constitutive law measurements through magnetic resonance imaging. Phys Rev E 78:036307

Ovarlez G, Barral Q, Coussot P (2010) Three-dimensional jamming and flows of soft glassy materials. Nat Mater 9:115-119

Ovarlez G, Mahaut F, Bertrand F, Chateau X (2011) Flows and heterogeneities with a vane tool: Magnetic resonance imaging measurements. J Rheol 5:197-223

Ovarlez G, Bertrand F, Coussot P, Chateau X (2012) Shear-induced sedimentation in yield stress fluids. J Non-Newtonian Fluid Mech 42:148-157

Pal R (2004) Rheological Constitutive Equation for Bubbly Suspensions. Ind Eng Chem Res 43:5372-5379

Parsi F, Gadala-Maria F (1987) Fore-and-aft asymmetry in a concentrated suspension of solid spheres. J Rheol 31:725-732

Phillips RJ, Armstrong RC, Brown RA, Graham AL, Abbott JR (1992) A constitutive equation for concentrated suspensions that accounts for shear-induced particle migration. Phys Fluids 4:30-40

Ramamurthy K, Kunhanandan Nambiar EK, Indu Siva Ranjani G (2009) A classification of studies on properties of foam concrete. Cement Concrete Comp 31:388-396

Rust AC, Manga M (2002a) Effects of bubble deformation on the viscosity of dilute suspensions. J Non-Newtonian Fluid Mech 104:53-63

Rust AC, Manga M (2002b) Bubble shapes and Orientations in low Re simple shear flow. J Colloid Interface Sci 249:476-480

Salonen A, Lhermerout R, Rio E, Langevin D, Saint-Jalmes A (2012) Dual gas and oil dispersions in water: production and stability of foamulsion. Soft Matter 8:699-706

Sikorski D, Tabuteau H, de Bruyn J (2009) Motion and shape of bubbles rising through a yield-stress fluid. J Non-Newtonian Fluid Mech 159:10-16

Stickel JJ, Powell RL (2005) Fluid Mechanics and Rheology of Dense Suspensions. Annu Rev Fluid Mech 37:129-149
Struble LJ, Jiang Q (2004) Effects of Air Entrainment on Rheology. Materials Journal 101:448-456

Turner D, Dlugogorski B, Palmer T (1999) Factors affecting the stability of foamed concentrated emulsions. Colloid Surface A 150:171184

Uhlerr PHT, Guo J, Tiu C, Zhang XM, Zhou JZQ, Fang TN (2005) The shear-induced solid-liquid transition in yield stress materials with chemically different structures. J Non-Newtonian Fluid Mech 125:101-119

van Aken GA (2001) Aeration of emulsions by whipping. Colloid Surface A 190:333-354

Vu TS, Ovarlez G, Chateau X (2010) Macroscopic behavior of bidisperse suspensions of noncolloidal particles in yield stress fluids. J Rheol 54:815-833 


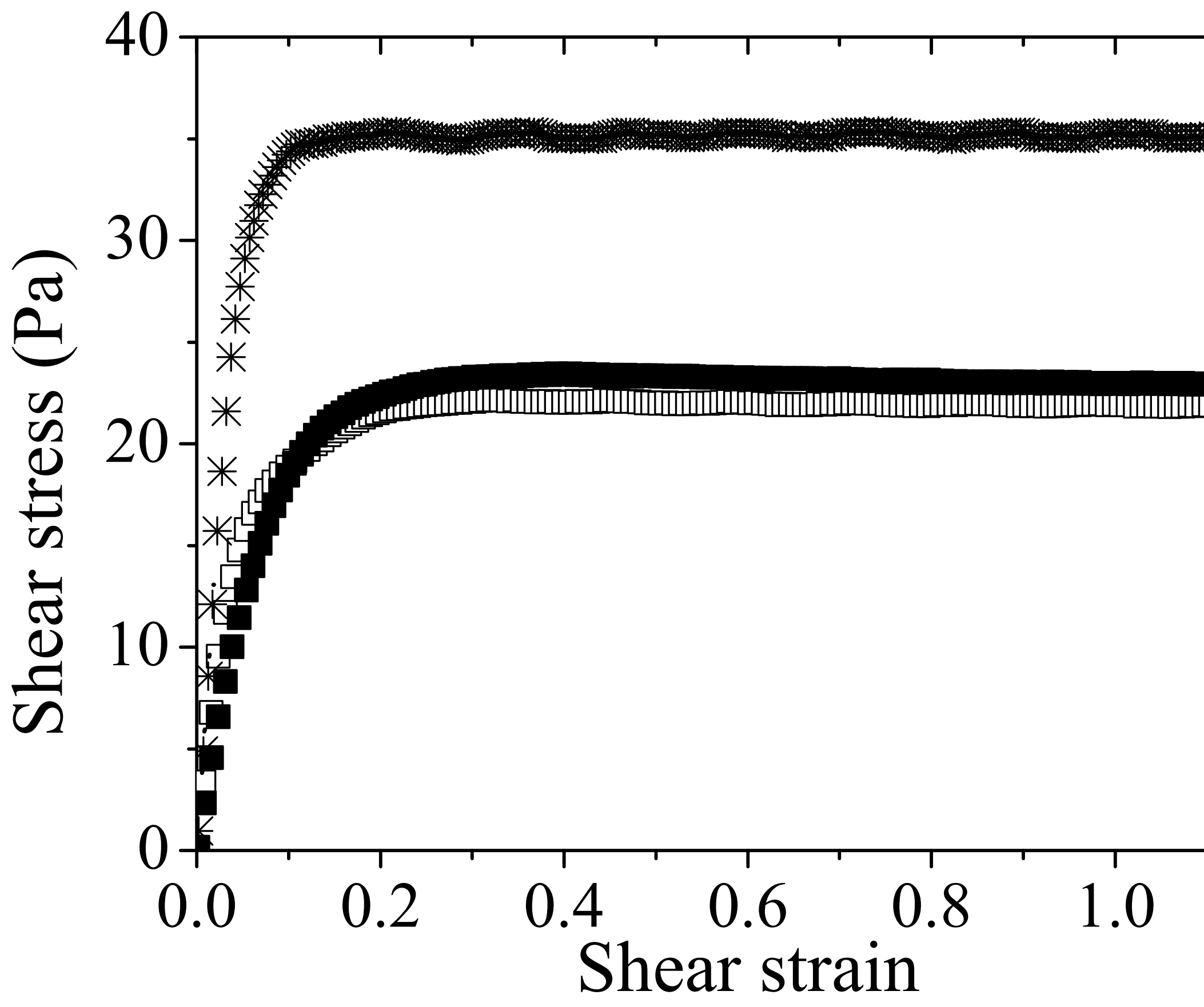

